\title{
Calorimetric and acoustic study of binary mixtures containing an isomeric chlorobutane and butyl ethyl ether or methyl tert-butyl ether
}

\author{
D. Montaño ${ }^{1}$, R. Bölts ${ }^{2}$, J. Gmehling ${ }^{2}$, H. Artigas ${ }^{3}$, C. Lafuente ${ }^{*, 3}$ \\ ${ }^{1}$ Departamento de Química, Facultad de Ciencias Exactas y Naturales, Universidad de \\ Antioquia, Medellín 1226, Colombia \\ 2 Departamento de Química Física, Facultad de Ciencias, Universidad de Zaragoza, \\ 50009 Zaragoza, Spain. \\ ${ }^{3}$ Technische Chemie, Universität Oldenburg, D-26111 Oldenburg, Germany. \\ *Corresponding author. Tel: +34 976762295, E-mail address: celadi@unizar.es
}

\begin{abstract}
Densities and speeds of sound in the temperature range $283.15 \mathrm{~K}$ to $313.15 \mathrm{~K}$ have been measured for the binary mixtures formed by an isomeric chlorobutane (1chlorobutane, 2-chlorobutane, 1-chloro-2-methylpropane, or 2-chloro-2-methylpropane) and butyl ethyl ether or methyl tert-butyl ether. Excess isentropic compressibilities were calculated from the experimental data. Excess enthalpies at $T=298.15 \mathrm{~K}$ are also included for the same binary mixtures. All these properties provide an insight into the nature of interactions operating on the present systems. Finally, the Prigogine-FloryPatterson theory has been used to analyze the $H^{\mathrm{E}}$ results and to estimate the isentropic compressibility values of the mixtures at $T=298.15 \mathrm{~K}$.
\end{abstract}

Keywords Chlorobutane isomers; Ethers; Excess enthalpies; Isentropic compressibility; Prigogine-Flory-Patterson theory. 


\section{Introduction}

Most of the industrial processes involve substances on liquid state, pure components or mixed systems with different concentrations of solvents. Detailed knowledge of the properties for this aggregation state of matter is a research area of permanent interest and continuous developments, as it demonstrates the constant appearance of new works on specialized publications. These works show two main and complementary routes in order to understand the molecular interactions that characterize this state. One route is the experimental way, trying to determine convenient properties as precise as possible in a wide range of conditions (composition, temperature, pressure...), and afterwards use the obtained values to derive new interesting properties by using reliable relationships (mainly thermophysical equations). The other main way lies on the formulation of theoretical models and correlations that could be used to predict the values of interesting properties in different conditions. Predicted values can be compared with experimental values in order to test their capability and validity.

The result of the researching group efforts is the enlargement of the available thermophysical property databases with always valuable experimental information. At the same time, this information is used to formulate more confidence and reliable predictive models. One way or another, the consequence is the better understanding of the processes that takes place in liquid substances and, especially, in their mixtures.

Continuing the study of mixtures involving chloroalkanes and ethers [1-6], we have chosen the binary systems formed by an isomer of chlorobutane (1-chlorobutane, 2-chlorobutane, 1-chloro-2-methylpropane, or 2-chloro-2-methylpropane) together with butyl ethyl ether or methyl tert-butyl ether. From experimental data of densities and speeds of sound along the temperature range of $283.15 \mathrm{~K}$ to $313.15 \mathrm{~K}$ excess isentropic compressibilities have been determined. This work also includes the excess enthalpies for the same systems at $T=298.15 \mathrm{~K}$ from which have been calculated. The obtained results have been compared with those previously reported for the systems isomer of chlorobutane with di-isopropyl ether [2-3].

Moreover, we have employed the Prigogine-Flory-Patterson theory to analyze the contributions to excess enthalpies [7-8]. Furthermore, we have also applied an extension of this theory to predict the isentropic compressibilities at $T=298.15 \mathrm{~K}$ from $H^{\mathrm{E}}$ values [9]. 


\section{Experimental}

The liquids used were: 1-chlorobutane, 2-chlorobutane, 2-chloro-2methylpropane, butyl ethyl ether and methyl tert-butyl ether, all of them provided by Sigma-Aldrich ( $\geq 99 \%$ ), and 1-chloro-2-methylpropane supplied by Fluka ( $>99 \%)$. After the confirmation of purities by gas chromatography no further purification was done.

The mixtures were prepared by mass using a Sartorious CP225-D semi-micro balance with an uncertainty of $\pm 10^{-5} \mathrm{~g}$. The maximum estimated error in the mole fraction is $\pm 1 \times 10^{-4}$.

Densities, $\rho$, and speeds of sound, $u$, of the samples, pure compounds and their mixtures, at atmospheric pressure were determined at the same time with an Anton Paar DSA-5000 vibrating tube densimeter and sound analyzer, internally thermostated within $\pm 0.001 \mathrm{~K}$. Being, the uncertainty of density measurements $\pm 5 \times 10^{-6} \mathrm{~g} \cdot \mathrm{cm}^{-3}$ and the uncertainty for speed of sound measurements $\pm 1 \times 10^{-1} \mathrm{~m} \cdot \mathrm{s}^{-1}$. The calibration was carried out with dry air and ultra pure water supplied by SH Calibrations Service $\mathrm{GmbH}$.

Excess enthalpies were obtained by means of a commercial flow calorimeter from Hart Scientific (model 7501), previously described [10]. In this device, a constant composition flow is passed through a thermostated calorimeter cell using two syringe pumps (model LC 2600, from ISCO). This cell is equipped with both a pulsed heater and a Peltier cooler that is working at constant power, producing a constant heat loss from the calorimeter cell, which is compensated by the pulsed heater. The heat effects influence the required frequency, so the heats of mixing can be determined from the observed frequency change with respect to the baseline. The evaporation and degassing effects are prevented using a back-pressure regulator. Finally, the temperatures are monitored with a Hart Scientific platinum resistance thermometer (model 1006 MicroTherm) with an uncertainty of $\pm 0.005 \mathrm{~K}$. The corresponding uncertainties in mole fraction and $H^{\mathrm{E}}$ were estimated to be \pm 0.0001 and less than $\pm 1 \%$ respectively. 


\section{Results and discussion}

From speeds of sound and densities the isentropic compressibilities can be estiamated by means of the relation: $\kappa_{\mathrm{S}}=1 /\left(\rho u^{2}\right)$, supposing that ultrasonic absorption is negligible. Then, excess isentropic compressibilities were estimated from these values using the following equations:

$$
\kappa_{\mathrm{S}}^{\mathrm{E}}=\kappa_{\mathrm{S}}-\kappa_{\mathrm{S}}^{\mathrm{id}}
$$

where $\kappa_{\mathrm{S}}^{\mathrm{id}}$ is calculated according to Benson and Kiyohara [11]:

$$
\kappa_{\mathrm{S}}^{\mathrm{id}}=\sum_{i} \phi_{\mathrm{i}}\left[\kappa_{\mathrm{S}, \mathrm{i}}+\frac{T V_{\mathrm{i}} \alpha_{\mathrm{p}, \mathrm{i}}^{2}}{C_{\mathrm{p}, \mathrm{i}}}\right]-T\left(\sum_{i} x_{\mathrm{i}} V_{\mathrm{i}}\right) \frac{\left(\sum_{i} \phi_{\mathrm{i}} \alpha_{\mathrm{p}, \mathrm{i}}\right)^{2}}{\left(\sum_{i} x_{\mathrm{i}} C_{\mathrm{p}, \mathrm{i}}\right)}
$$

being, respectively, $\phi_{\mathrm{i}}$ the volume fraction of component $i$ in the mixture referred to the unmixed state, $x_{\mathrm{i}}$ the corresponding mole fraction, $T$ the temperature, and $\kappa_{\mathrm{S}, \mathrm{i}}, V_{\mathrm{i}}, \alpha_{\mathrm{p}, \mathrm{i}}$ $C_{\mathrm{p}, \mathrm{i}}$ the isentropic compressibility, the molar volume, the isobaric expansibilities, and the isobaric molar heat capacity of the pure component $i$.

Isobaric expansibilities, $\alpha_{\mathrm{p}}$, were derived from densities measured in our laboratory. Isobaric molar heat capacities for 1-chlorobutane and methyl tert-butyl ether were taken from the literature $[12,13]$ and for the rest of compounds were calculated using group-contribution methods $[14,15]$. The pure compound properties are listed in Table 1.

Excess isentropic compressibilities are graphically shown in Figs. 1 to 8 .

Excess enthalpies for all the mixtures were obtained from thermal energy per time unit data. This property is drawn on Figs. 9 and 10.

The values of each excess property, $Y^{\mathrm{E}}$, were correlated with a Redlich-Kister polynomial expansion, $A_{\mathrm{i}}$ are adjustable parameters and $x_{\mathrm{i}}$ is the mole fraction of component $i$.

$$
Y^{\mathrm{E}}=x_{1} x_{2} \sum_{i} A_{\mathrm{i}}\left(x_{1}-x_{2}\right)^{i}
$$


The values of the parameters $A_{\mathrm{i}}$, together with the standard deviations $\sigma\left(Y^{\mathrm{E}}\right)$ are given in Table 2

The measured properties (densities and speeds of sound) together with the excess properties can be found in the supplementary information.

Excess isentropic compressibilities are negative over the whole composition range at all temperatures, and they become more negative when temperature increases. For a given chlorobutane, the values are lower in mixtures containing methyl tert-butyl ether. If we consider mixtures with the same ether, the most negative values correspond to systems with the chlorine atom bonded to a primary carbon (1-chlorobutane and 1chloro-2-methylpropane) and the less negative ones correspond to the other two isomers. It is remarkably that values with 2-chloro-2-methylpropane are similar independently of the ether involved, but the system 2-chlorobutane + butyl ethyl ether presents the highest values of all here studied (minimum values of -3.7 and $-4.8 \mathrm{TPa}^{-1}$ ). This comment can be also done regarding mixtures with 1-chloro-2-methylpropane, because they present almost similar values independently of the ether considered. Then, we can conclude that values for this property are highly influenced by the branched structure of the chlorinated compound. Nevertheless, minimum values are always placed at molar fractions equal to 0.5 .

If we compare the results here presented with those corresponding to systems formed by the four chlorobutanes and diisopropyl ether [2] the only difference is that the values for these last systems are more negative except for the mixture with 2-chloro2-methylpropane that again presents values almost similar to the systems with the other two ethers.

Thermal behaviour at $T=298.15 \mathrm{~K}$ is very different for the mixtures depending on the ether considered. $H^{E}$ values are negative for mixtures with methyl tert-butyl ether (minimum values vary in the range -90 and $-220 \mathrm{~kJ} \cdot \mathrm{mol}^{-1}$ ), but they are positive for the systems 2-chlorobutane or 2-chloro-2-methylpropane + butyl ethyl ether $\left(70 \mathrm{~kJ} \cdot \mathrm{mol}^{-1}\right.$ in the maxima), and S-shaped for mixtures with primary chlorinated isomers (1chlorobutane and 1-chloro-2-methylpropane) In these cases, values are negative in the region richest in ether (until molar fraction 0.6) and positive in the rest. Nevertheless, values are always near zero, varying between -10 and $15 \mathrm{~kJ} \cdot \mathrm{mol}^{-1}$. If we consider the same ether, values of this property follow the sequence: 
2-chloro-2-methylpropane $>$ 2-chlorobutane $>$ 1-chlorobutane $>$ 1-chloro-2methylpropane.

Systems containing diisopropyl ether with the same haloalkanes follows the same trend, being values with 2-chloro-2-methylpropane positive and small, and somewhat negative for the rest. Minimum excess enthalpies are virtually similar for systems with 1-chlorobutane and 1-chloro-2-methylpropane, and lower (absolute terms) when haloalkane is 2-chlorobutane [3].

These values can be interpreted as the result of the forces operating in pure liquids and the mixture. All substances here used have moderate dipole moment, between 1.90 and 2.15 D for chlorinated isomers [16] and 1.24 and 1.225 D for butyl ethyl ether and methyl tert-butyl ether respectively [16,17]. Then, it can be expected dipole-dipole interactions as responsible of the observed behaviour. Besides, ethers present an oxygen atom with two electron pairs that can play as electron density donor [18], meanwhile chlorine atom of chlorobutanes presents a positive electrostatic potential region outside the molecule. The result is an electrostatically driven non-covalent interaction $[19,20]$ established between ether and haloalkane molecules. When mixing, the opposite energetic interactions operates together, mitigating like-like interactions and establishing new $\mathrm{Cl}-\mathrm{O}$ interaction corresponding to the unlike substances.

Negative excess enthalpies for the mixtures containing methyl tert-butyl ether indicate that $\mathrm{Cl}-\mathrm{O}$ interaction contribution plays an important role in the mixing process, compensating the weakening of the dipole-dipole existing in the pure compounds. The position of the $\mathrm{Cl}$ atom is also an important factor, being the most exposed (primary haloalkanes) those with the most negative values due to their easiest possibility to establish the interaction. In the case of the systems haloalkane + butyl ethyl ether, linear structure of both hydrocarbon chains bonded by $\mathrm{O}$ atom seems to hinder $\mathrm{Cl}-\mathrm{O}$ interaction, doing this interaction only noticeable with primary chlorinated compounds.

This interpretation can be confirmed regarding excess isentropic compressibility values, property more related with structural effects. The values of this property are clearly more negative for systems with methyl tert-butyl ether than those with butyl ethyl ether, resulting in more compact systems. The accommodation between structures seems to be important with the first cited ether, with almost spherical hydrocarbon chains around $\mathrm{O}$ atom compared with linear ones for butyl ethyl ether. This fact, combined with the contribution of the $\mathrm{Cl}-\mathrm{O}$ interaction could explain the negative values for this property in the four systems with methyl tert-butyl ether and only with 
the two primary chlorinated hydrocarbons in the mixtures with butyl ethyl ether.

\section{Prigogine-Flory-Patterson theory}

In the Prigogine-Flory-Patterson theory the excess enthalpy of a mixture can be expressed as the sum of two contribution [7, 8]: an interactional contribution which is proportional to the Flory or interaction parameter, $\chi_{12}$, and a free volume contribution:

$$
\frac{H^{\mathrm{E}}}{x_{1} U_{1}^{*}+x_{2} U_{2}^{*}}=\frac{\left[-\widetilde{U}+\widetilde{T} \widetilde{C}_{\mathrm{p}}\right] \psi_{1} \theta_{2} \chi_{12}}{P_{1}^{*}}+\widetilde{C}_{\mathrm{p}}\left[\psi_{1} \widetilde{T}_{1}+\psi_{2} \widetilde{T}_{2}-\widetilde{T}\right]
$$

In this equation the reduced magnitudes for the mixture $\left(\widetilde{V}, \widetilde{T}\right.$, and $\left.\widetilde{C}_{\mathrm{p}}\right)$ can be obtained from the reduced configurational energy, $\widetilde{U}$, which was calculated using the reduced configurational energies of the pure components by:

$$
\widetilde{U}=\psi_{1} \widetilde{U}_{1}+\psi_{2} \widetilde{U}_{2}
$$

where the contact energy fraction, $\psi_{\mathrm{i}}$, is defined as:

$$
\psi_{1}=1-\psi_{2}=\frac{x_{1} P_{1}^{*} V_{1}^{*}}{x_{1} P_{1}^{*} V_{1}^{*}+x_{2} P_{2}^{*} V_{2}^{*}}
$$

The rest of the parameters can be obtained using Flory's theory [21, 22].

The Prigogine-Flory-Patterson theory was extended by Oswall [9] in order to estimate the isentropic compressibilities of liquid mixtures. Once the interaction parameter, $\chi_{12}$, is obtained the Prigogine-Flory-Patterson theory allows to estimate the molar volumes, $V$, the molar heat capacities, $C_{\mathrm{p}}$, and the coefficients $(\partial V / \partial T)_{p}$ and $(\partial V / \partial p)_{T}$ of a liquid mixture. From these quantities, by using a thermodynamic relation the isentropic compressibility, $\kappa_{S}=-V^{-1}(\partial V / \partial p)_{S}$, can be obtained: 


$$
(\partial V / \partial p)_{S}=(\partial V / \partial p)_{T}+T C_{\mathrm{p}}^{-1}(\partial V / \partial T)_{p}^{2}
$$

Physical properties of the pure compounds together with Flory parameters are given in Table 3. Isothermal compressibilities, $\kappa_{\mathrm{T}}$, were calculated from volumetric behaviour $(p \rho T)$ and the number of contact sites per segment of a molecule, $s$, has been estimated using the Bondi's method [23].

Here, we have obtained the interaction parameter for each mixture by fitting the Prigogine-Flory-Patterson theory to the corresponding experimental equimolar $H^{\mathrm{E}}$ values. Table 4 provides the interaction parameter for each system, the calculated contributions to $H^{\mathrm{E}}$ together with the estimated and experimental $\kappa_{\mathrm{S}}$ values at equimolar composition and at $T=298.15 \mathrm{~K}$.

For all the systems the interactional contribution is very small in absolute value and negative except for the mixtures 2-chlorobutane + butyl ethyl ether and 1-chloro-2methylpropane for which this contribution equals to zero. On the other hand the free volume contribution is the dominant term for all the mixtures being this contribution positive for the systems presenting positive $H^{\mathrm{E}}$ values that is, 2-chlorobutane + butyl ethyl ether and 2-chloro-2-methylpropane + butyl ethyl ether. With respect to the prediction of isentropic compressibilities, it can be considered satisfactory with an overall deviation from experimental results less than $1.8 \%$, the best predictions correspond to the mixtures containing 1-chlorobutane while the worst predictions are found for the mixtures containing 1-chloro-2-methylpropane. It can be outlined that for the systems studied here the predicted $\kappa_{\mathrm{S}}$ values are slightly lower than the experimental ones.

\section{Conclusions}

The measurement of density and speed of sound over the whole composition range for the binary mixtures of an isomer of chlorobutane and butyl ethyl ether or methyl tert-butyl ether at different temperatures (from 283.15 to $313.15 \mathrm{~K}$ ) has allowed the determination of the isentropic compressibilities and the calculation of the excess isentropic compressibilities of these systems. This excess property exhibits negative 
values in all cases, being the lowest those corresponding to the systems 1-chlorobutane or 1-chloro-2-methylpropane at the highest temperature. Excess enthalpies for the same mixtures have been determined at $T=298.15 \mathrm{~K}$, showing negative values all systems formed by an isomer of chlorobutane with methyl tert-butyl ether, and mainly positive with butyl ethyl ether.

Considering these results, $\mathrm{Cl}-\mathrm{O}$ interaction established in the mixing process seems to be the main responsible of the observed behaviour, combined with structural effects due to the different shape of the alkyl chains of the ethers.

Prigogine-Flory-Patterson theory has been applied to analyze excess enthalpies in order to obtain interaction parameter to derive isentropic compressibilities in order to compare with experimental values. The results are satisfactory, with an overall deviation less than $1.8 \%$.

Acknowledgements Authors are indebted to Diputación General de Aragón and Fondo Social Europeo “Construyendo Europa desde Aragón”. 


\section{References}

[1] Giner I, Villares A, Haro M, Gascón I, Lafuente C. Thermodynamic properties of binary mixtures formed by cyclic ethers and chloroalkanes. J Therm Anal Calorim. 2007;90:9587-595.

[2] Montaño DF, Martín S, Cea P, López MC, Artigas H. Volumetric, acoustic, and refractive properties of isomeric chlorobutanes with diisopropyl ether. J Chem Eng Data. 2010;55:5953-5959.

[3] Montaño D, Gascón I, Bölts R, Gmehling J, Lafuente C. Isothermal vapourliquid equilibria and excess enthalpies for the binary mixtures containing an isomeric chlorobutane and diisopropyl ether. Fluid Phase Equilib. 2011;308:814.

[4] Montaño D, Gascón I, Schmid B, Gmehling J, Lafuente C. Simultaneous prediction of densities and vapour-liquid equilibria of mixtures containing an isomeric chlorobutane and methyl tert-butyl ether using the VTPR model. Ind Eng Chem Res. 2011;50:14193-14202.

[5] Montaño D, Gascón I, Schmid B, Gmehling J, Lafuente C. Experimental and predicted properties of the binary mixtures containing an isomeric chlorobutane and butyl ethyl ether. J Chem Thermodyn. 2012;51:150-158.

[6] Montaño D, Giner B, Martín S, Lafuente C. Surface study of binary mixtures containing chlorinated and oxygenated compounds. J Mol Liq. 2013;18:1-7.

[7] Van HT, Patterson D. Volumes of mixing and the P* effect. 1. Hexane isomers with normal and branched hexadecane. J Solution Chem. 1982;11:793-805.

[8] Costas M, Patterson D. Volumes of mixing and the P* effect. 2. Mixtures of alkanes with liquids of different internal pressures. J Solution Chem 1982;11:807-821.

[9] Oswal SL. Theoretical estimation of isentropic compressibility and speed of sound in binary liquid mixtures from the Prigogine-Flory-Patterson theory. Acoustics Lett. 1990;14:17-25.

[10] Gmehling J. Excess enthalpies for 1,1,1-trichloroethane with alkanes, ketones, and esters, J Chem Eng Data, 1993;38:143-146.

[11] Benson GC, Kiyohara O. Evaluation of excess isentropic compressibilities and isochoric heat-capacities. J Chem Thermodyn. 1979;11:1061-1064. 
[12] Chorazewski M, Goralski P, Tkaczyk M. Heat capacities of 1-chloroalkanes and 1-bromoalkanes within the temperature range from $284.15 \mathrm{~K}$ to $353.15 \mathrm{~K}$. A group additivity and molecular connectivity analysis. J Chem Eng Data, 2005;50:619-624.

[13] Andon RJL, Martin JF. Thermodynamic properties of organic oxygen compounds .40. Heat-capacity and entropy of 6 ethers. J Chem Thermodyn, 1975;7:593-606.

[14] Ruzicka V, Domalski ES. Estimation of the heat capacities of organic liquids as a function of temperature using group additivity. 1. Hydrocarbon compounds. J Phys Chem Ref Data, 1993;22:597-618.

[15] Kolska Z. Kukal J, Zabransk M, Ruzicka V. Estimation of the heat capacity of organic liquids as a function of temperature by a three-level group contribution method. Ind Eng Chem Res, 2008;47:2075-2085.

[16] Riddick JA, Bunger WB, Sakano TK, Organic Solvents. Physical Properties and Methods of Purification, $4^{\text {th }}$ ed; Wiley-Interscience, New York 1986.

[17] Suenram RD, Lovas FJ, Pereyra W, Fraser GT, Walker ARH. Rotational spectra, structure, and electric dipole moments of methyl and ethyl tert-butyl ether (MTBE and ETBE). J Mol Spectrosc. 1997;181:67-77.

[18] Arnett EM, Wu CY. Base strenghts of some aliphatic ethers in aqueous sulfuric acid. J Am Chem Soc. 1962;84:1680-1684.

[19] Marongiu B, Porcedda S. Excess enthalpies of some binary mixtures: a contribution to the study of the $\mathrm{Cl}-\mathrm{O}$ specific interaction. Thermochim Acta. 1989; 155:317-325.

[20] Politzer P, Murray JS, Clark T. Halogen bonding: an electrostatically-driven highly directional noncovalent interaction. Phys Chem Chem Phys. 2010;12:7748-7757.

[21] Flory PJ, Orwoll RA, Vrij A. Statistical thermodynamics of chain molecule liquids. I. Equation of state for normal paraffin hydrocarbons. J Am Chem Soc. 1964;86:3507-3514.

[22] Abe A, Flory PJ. Thermodynamics properties of mixtures of small nonpolar molecules. J Am Chem Soc. 1965;87:1838-1846.

[23] Bondi A. Van der Waals volumes + radii. J Phys Chem. 1964;68:441-451. 
Table 1 Densities, $\rho$, speeds of sound, $u$, isobaric expansibilities, $\alpha_{\mathrm{p}}$, and molar heat capacities, $C_{\mathrm{p}}$ of pure components at working temperatures.

\begin{tabular}{|c|c|c|c|c|c|}
\hline Compound & $T / \mathrm{K}$ & $\rho / \mathrm{g} \cdot \mathrm{cm}^{-3}$ & $u / \mathrm{m} \cdot \mathrm{s}^{-1}$ & $\alpha_{\mathrm{p}} / \mathrm{kK}^{-1}$ & $\begin{array}{c}C_{\mathrm{p}} / \\
\mathrm{J} \cdot \mathrm{K}^{-1} \cdot \mathrm{mol}^{-1}\end{array}$ \\
\hline \multirow{7}{*}{ 1-Chlorobutane } & 283.15 & 0.897339 & 1180.14 & 1.2210 & 155.96 \\
\hline & 288.15 & 0.891855 & 1159.51 & 1.2350 & 157.06 \\
\hline & 293.15 & 0.886335 & 1138.84 & 1.2490 & 158.10 \\
\hline & 298.15 & 0.880705 & 1118.18 & 1.2640 & 159.16 \\
\hline & 303.15 & 0.875204 & 1097.64 & 1.2797 & 160.17 \\
\hline & 308.15 & 0.869585 & 1077.18 & 1.2960 & 161.44 \\
\hline & 313.15 & 0.863882 & 1056.91 & 1.3130 & 162.65 \\
\hline \multirow{7}{*}{ 2-Chlorobutane } & 283.15 & 0.884340 & 1127.71 & 1.2586 & 156.16 \\
\hline & 288.15 & 0.878756 & 1107.25 & 1.2756 & 157.29 \\
\hline & 293.15 & 0.873130 & 1086.74 & 1.2921 & 158.40 \\
\hline & 298.15 & 0.867474 & 1066.24 & 1.3087 & 159.49 \\
\hline & 303.15 & 0.861779 & 1045.88 & 1.3260 & 160.58 \\
\hline & 308.15 & 0.856044 & 1025.33 & 1.3446 & 161.65 \\
\hline & 313.15 & 0.850266 & 1005.32 & 1.3652 & 162.70 \\
\hline \multirow{7}{*}{ 1-Chloro-2-methylpropane } & 283.15 & 0.888845 & 1141.74 & 1.2656 & 156.78 \\
\hline & 288.15 & 0.883211 & 1120.72 & 1.2813 & 158.03 \\
\hline & 293.15 & 0.877539 & 1099.63 & 1.2966 & 159.31 \\
\hline & 298.15 & 0.871533 & 1078.68 & 1.3123 & 160.63 \\
\hline & 303.15 & 0.866096 & 1057.76 & 1.3292 & 161.98 \\
\hline & 308.15 & 0.860319 & 1036.97 & 1.3474 & 163.36 \\
\hline & 313.15 & 0.853902 & 1016.27 & 1.3667 & 164.78 \\
\hline \multirow{7}{*}{ 2-Chloro-2-methylpropane } & 283.15 & 0.855249 & 1046.93 & 1.3990 & 155.76 \\
\hline & 288.15 & 0.849243 & 1025.50 & 1.4200 & 157.75 \\
\hline & 293.15 & 0.843191 & 1004.08 & 1.4406 & 159.78 \\
\hline & 298.15 & 0.837096 & 983.25 & 1.4614 & 161.86 \\
\hline & 303.15 & 0.830958 & 961.51 & 1.4829 & 163.98 \\
\hline & 308.15 & 0.824772 & 940.46 & 1.5058 & 166.16 \\
\hline & 313.15 & 0.818536 & 919.41 & 1.5307 & 168.37 \\
\hline \multirow{7}{*}{ Butyl ethyl ether } & 283.15 & 0.759632 & 1159.20 & 1.2562 & 219.24 \\
\hline & 288.15 & 0.754838 & 1137.83 & 1.2726 & 220.52 \\
\hline & 293.15 & 0.750018 & 1116.61 & 1.2889 & 221.82 \\
\hline & 298.15 & 0.745173 & 1095.40 & 1.3054 & 223.14 \\
\hline & 303.15 & 0.740300 & 1074.35 & 1.3218 & 224.47 \\
\hline & 308.15 & 0.735393 & 1053.45 & 1.3382 & 225.83 \\
\hline & 313.15 & 0.730451 & 1012.18 & 1.3546 & 227.20 \\
\hline
\end{tabular}


Table 1 Continuation.

\begin{tabular}{cccccc}
\hline Compound & $T / \mathrm{K}$ & $\rho / \mathrm{g} \cdot \mathrm{cm}^{-3}$ & $u / \mathrm{m} \cdot \mathrm{s}^{-1}$ & $\alpha_{\mathrm{p}} / \mathrm{mK}^{-1}$ & $\begin{array}{c}C_{\mathrm{P}, \mathrm{m}} / \\
\mathrm{J} \cdot \mathrm{K}^{-1} \cdot \mathrm{mol}^{-1}\end{array}$ \\
\hline \multirow{5}{*}{ Methyl tert-butyl ether } & 283.15 & 0.750938 & 1105.89 & 1.3654 & 182.70 \\
& 288.15 & 0.745787 & 1082.66 & 1.3882 & 184.27 \\
& 293.15 & 0.740585 & 1059.49 & 1.4109 & 185.87 \\
& 298.15 & 0.735337 & 1036.25 & 1.4340 & 187.50 \\
& 303.15 & 0.730042 & 1013.17 & 1.4578 & 189.16 \\
& 308.15 & 0.724693 & 990.25 & 1.4829 & 190.86 \\
& 313.15 & 0.719293 & 967.35 & 1.5098 & 192.58 \\
\hline
\end{tabular}


Table 2 Parameters, $A_{\mathrm{i}}$, and standard deviations, $\sigma\left(Y^{\mathrm{E}}\right)$, for Redlich-Kister's equation.

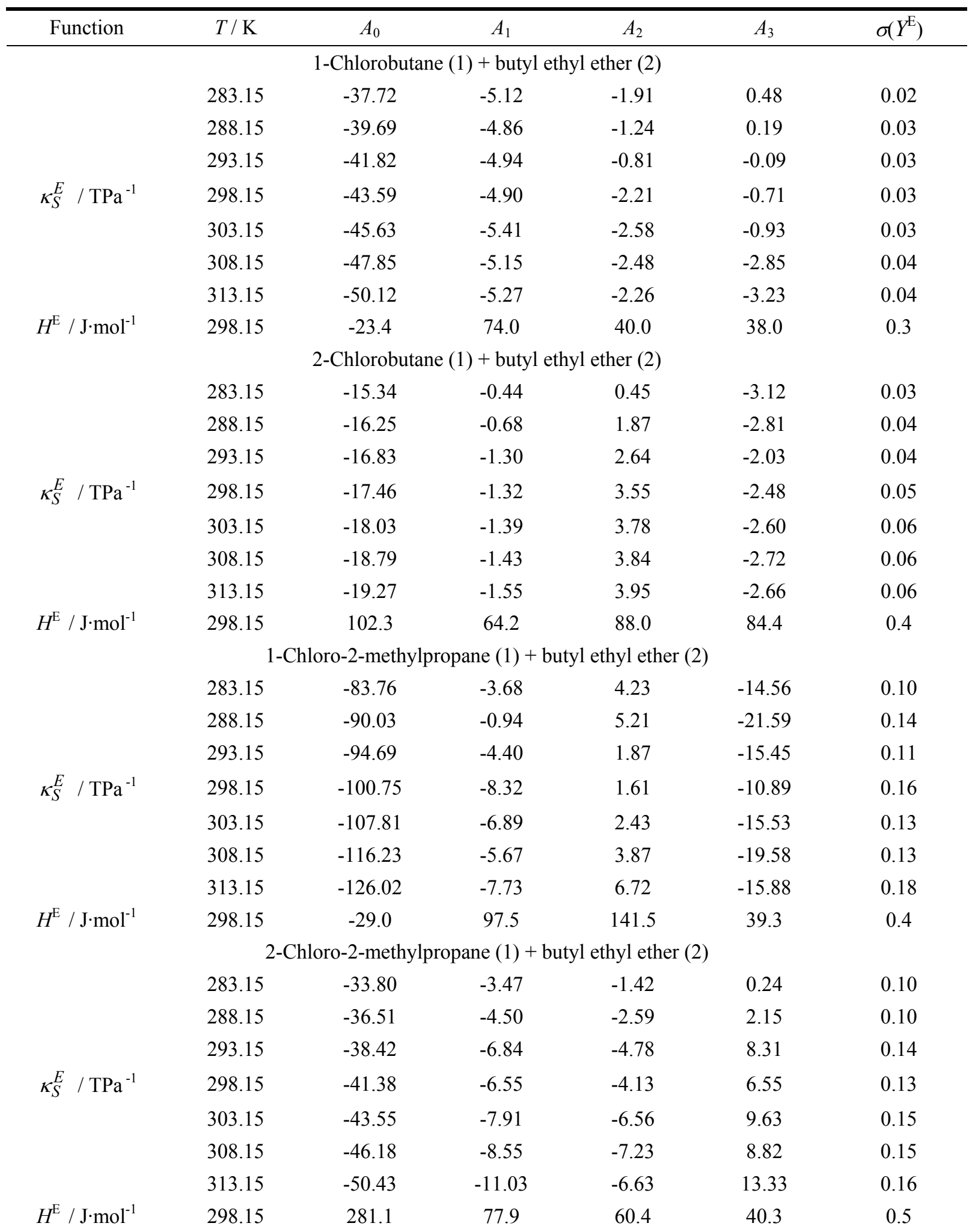


Table 2 Continuation.

\begin{tabular}{|c|c|c|c|c|c|c|}
\hline Function & $T / \mathrm{K}$ & $A_{0}$ & $A_{1}$ & $A_{2}$ & $A_{3}$ & $\sigma\left(Y^{\mathrm{E}}\right)$ \\
\hline \multicolumn{7}{|c|}{ 1-Chlorobutane (1) + methyl tert-butyl ether (2) } \\
\hline & 283.15 & -107.22 & 5.79 & -2.13 & 2.53 & 0.09 \\
\hline & 288.15 & -116.57 & 7.10 & -3.32 & 4.20 & 0.16 \\
\hline & 293.15 & -126.53 & 6.28 & -2.74 & 2.52 & 0.11 \\
\hline \multirow[t]{4}{*}{$\kappa_{S}^{E} / \mathrm{TPa}^{-1}$} & 298.15 & -138.26 & 7.66 & -4.03 & 3.98 & 0.11 \\
\hline & 303.15 & -151.65 & 8.74 & -2.53 & 3.36 & 0.12 \\
\hline & 308.15 & -165.77 & 8.51 & -3.01 & 6.62 & 0.11 \\
\hline & 313.15 & -181.96 & 10.95 & -4.49 & 5.26 & 0.15 \\
\hline$H^{\mathrm{E}} / \mathrm{J} \cdot \mathrm{mol}^{-1}$ & 298.15 & -717.4 & 24.9 & 18.3 & -25.8 & 1.0 \\
\hline \multicolumn{7}{|c|}{ 2-Chlorobutane (1) + methyl tert-butyl ether (2) } \\
\hline \multirow{7}{*}{$\kappa_{S}^{E} / \mathrm{TPa}^{-1}$} & 283.15 & -62.65 & 0.68 & -2.52 & 6.67 & 0.09 \\
\hline & 288.15 & -68.17 & 1.48 & -0.63 & 6.00 & 0.10 \\
\hline & 293.15 & -73.87 & 2.02 & -4.03 & 7.95 & 0.10 \\
\hline & 298.15 & -81.00 & 1.38 & -1.54 & 7.93 & 0.13 \\
\hline & 303.15 & -88.22 & 1.72 & -1.65 & 7.85 & 0.14 \\
\hline & 308.15 & -96.32 & 2.38 & -1.28 & 7.28 & 0.15 \\
\hline & 313.15 & -106.02 & 2.63 & 0.20 & 6.25 & 0.12 \\
\hline \multirow[t]{5}{*}{$H^{\mathrm{E}} / \mathrm{J} \cdot \mathrm{mol}^{-1}$} & 298.15 & -536.8 & -4.1 & 9.3 & -4.5 & 0.6 \\
\hline & \multicolumn{5}{|c|}{ 1-Chloro-2-methylpropane (1) + methyl tert-butyl ether (2) } & \\
\hline & 283.15 & -79.44 & -3.03 & 2.99 & 15.30 & 0.12 \\
\hline & 288.15 & -86.58 & -6.10 & 3.99 & 22.28 & 0.15 \\
\hline & 293.15 & -92.29 & -2.91 & 0.67 & 15.96 & 0.13 \\
\hline \multirow[t]{4}{*}{$\kappa_{S}^{E} / \mathrm{TPa}^{-1}$} & 298.15 & -99.62 & 0.72 & 0.26 & 11.43 & 0.15 \\
\hline & 303.15 & -108.04 & -1.26 & 1.03 & 16.34 & 0.14 \\
\hline & 308.15 & -117.83 & -2.96 & 2.11 & 20.53 & 0.18 \\
\hline & 313.15 & -129.66 & -1.27 & 4.76 & 16.76 & 0.20 \\
\hline \multirow[t]{5}{*}{$H^{\mathrm{E}} / \mathrm{J} \cdot \mathrm{mol}^{-1}$} & 298.15 & -845.9 & 25.5 & 10.5 & -15.8 & 0.6 \\
\hline & \multicolumn{5}{|c|}{ 2-Chloro-2-methylpropane (1) + methyl tert-butyl ether (2) } & \\
\hline & 283.15 & -32.14 & 2.03 & -5.08 & 3.70 & 0.06 \\
\hline & 288.15 & -34.05 & 1.04 & -4.79 & 6.16 & 0.08 \\
\hline & 293.15 & -35.87 & 1.15 & -6.21 & 5.15 & 0.08 \\
\hline \multirow[t]{4}{*}{$\kappa_{S}^{E} / \mathrm{TPa}^{-1}$} & 298.15 & -37.70 & -0.85 & -8.02 & 10.41 & 0.08 \\
\hline & 303.15 & -40.37 & -0.73 & -7.27 & 11.74 & 0.09 \\
\hline & 308.15 & -42.61 & -1.05 & -7.53 & 11.53 & 0.07 \\
\hline & 313.15 & -45.29 & -3.01 & -10.71 & 13.65 & 0.09 \\
\hline$H^{\mathrm{E}} / \mathrm{J} \cdot \mathrm{mol}^{-1}$ & 298.15 & -380.2 & -19.9 & 3.8 & -22.3 & 0.8 \\
\hline
\end{tabular}


Table 3 Physical properties and Flory parameters of the pure compounds at $T=298.15 \mathrm{~K}$

\begin{tabular}{cccccc}
\hline Compound & $\alpha_{\mathrm{p}} / \mathrm{kK}^{-1}$ & $\kappa_{\mathrm{T}} / \mathrm{TPa}^{-1}$ & $P^{*} / \mathrm{J} \cdot \mathrm{cm}^{-3}$ & $\widetilde{V}$ & $s / \AA^{-1}$ \\
\hline 1-Chlorobutane & 1.2640 & 1232.68 & 516.20 & 1.2994 & 1.424 \\
2-Chlorobutane & 1.3087 & 1366.07 & 488.52 & 1.3078 & 1.410 \\
1-Chloro-2-methylpropane & 1.3123 & 1341.73 & 499.21 & 1.3084 & 1.422 \\
2-Chloro-2-methylpropane & 1.4614 & 1675.35 & 463.65 & 1.3352 & 1.446 \\
Butyl ethyl ether & 1.3054 & 1439.60 & 461.91 & 1.3071 & 1.423 \\
Methyl tert-butyl ether & 1.4340 & 1677.26 & 451.18 & 1.3304 & 1.471 \\
\hline
\end{tabular}


Table 4 Interaction parameter, $\chi_{12}$, calculated $H^{\mathrm{E}}$ contributions and experimental and calculated $\kappa_{\mathrm{S}}$ at equimolar composition and at $T=298.15 \mathrm{~K}$.

\begin{tabular}{|c|c|c|c|c|c|}
\hline \multirow{2}{*}{$H^{\mathrm{E}} / \mathrm{J} \cdot \mathrm{mol}^{-1}$} & \multirow{2}{*}{$\chi_{12} / \mathrm{J} \cdot \mathrm{cm}^{-3}$} & \multicolumn{2}{|c|}{$H^{\mathrm{E}}$ contributions $/ \mathrm{J} \cdot \mathrm{mol}^{-1}$} & \multicolumn{2}{|c|}{$\kappa_{\mathrm{S}} / \mathrm{TPa}^{-1}$} \\
\hline & & Interactional & Free volume & Exptl. & PFP \\
\hline \multicolumn{6}{|c|}{ 1-Chlorobutane (1) + butyl ethyl ether (2) } \\
\hline-5.9 & -0.23 & -0.3 & -5.6 & 1016.4 & 1029.2 \\
\hline \multicolumn{6}{|c|}{ 2-Chlorobutane (1) + butyl ethyl ether (2) } \\
\hline 25.6 & 1.04 & 0.0 & 25.6 & 1069.1 & 1082.7 \\
\hline \multicolumn{6}{|c|}{ 1-Chloro-2-methylpropane (1) + butyl ethyl ether (2) } \\
\hline-7.3 & -0.30 & 0.0 & -7.3 & 1036.1 & 1071.2 \\
\hline \multicolumn{6}{|c|}{ 2-Chloro-2-methylpropane (1) + butyl ethyl ether (2) } \\
\hline 70.3 & 3.00 & -3.4 & 73.7 & 1164.6 & 1180.4 \\
\hline \multicolumn{6}{|c|}{ 1-Chlorobutane (1) + methyl tert-butyl ether (2) } \\
\hline-179.4 & -7.60 & -4.1 & -175.3 & 1065.0 & 1083.2 \\
\hline \multicolumn{6}{|c|}{ 2-Chlorobutane (1) + methyl tert-butyl ether (2) } \\
\hline-134.2 & -5.66 & -2.1 & -132.1 & 1127.3 & 1146.8 \\
\hline \multicolumn{6}{|c|}{ 1-Chloro-2-methylpropane (1) + methyl tert-butyl ether (2) } \\
\hline-211.5 & -9.04 & -2.0 & -209.5 & 1110.1 & 1131.5 \\
\hline \multicolumn{6}{|c|}{ 2-Chloro-2-methylpropane (1) + methyl tert-butyl ether (2) } \\
\hline-95.1 & -4.07 & -0.1 & -95.0 & 1242.8 & 1261.2 \\
\hline
\end{tabular}


Fig. 1

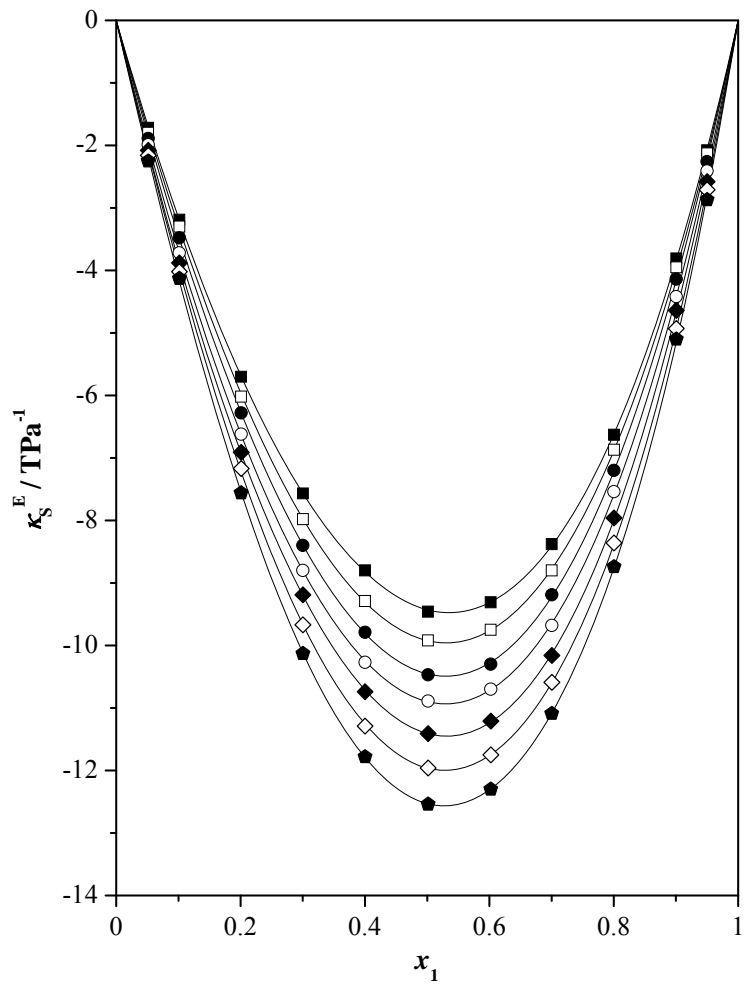

Excess isentropic compressibilities, $\kappa_{S}^{E}$, for 1-chlorobutane + butyl ethyl ether as a function of mole fraction, $x_{1}:(\square) T=283.15 \mathrm{~K},(\square) T=288.15 \mathrm{~K},(\bullet) T=293.15 \mathrm{~K},(\bigcirc) T=298.15 \mathrm{~K}$, $(\diamond) T=303.15 \mathrm{~K},(\diamond) T=308.15 \mathrm{~K},(\bullet) T=313.15 \mathrm{~K}$; $(-)$ Redlich-Kister equation. 
Fig. 2

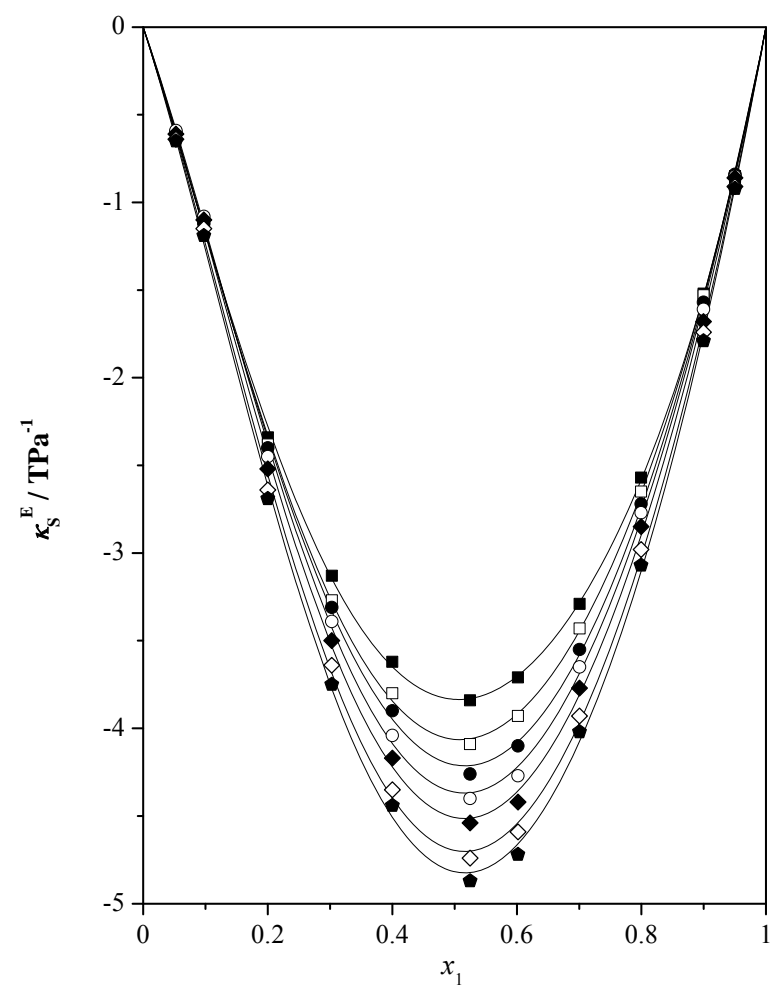

Excess isentropic compressibilities, $\kappa_{S}^{E}$, for 2-chlorobutane + butyl ethyl ether as a function of mole fraction, $x_{1}:(\square) T=283.15 \mathrm{~K},(\square) T=288.15 \mathrm{~K}$, () $T=293.15 \mathrm{~K}$, (○) $T=298.15 \mathrm{~K}$, $(\diamond) T=303.15 \mathrm{~K},(\diamond) T=308.15 \mathrm{~K},(\bullet) T=313.15 \mathrm{~K}$; $(-)$ Redlich-Kister equation. 
Fig. 3

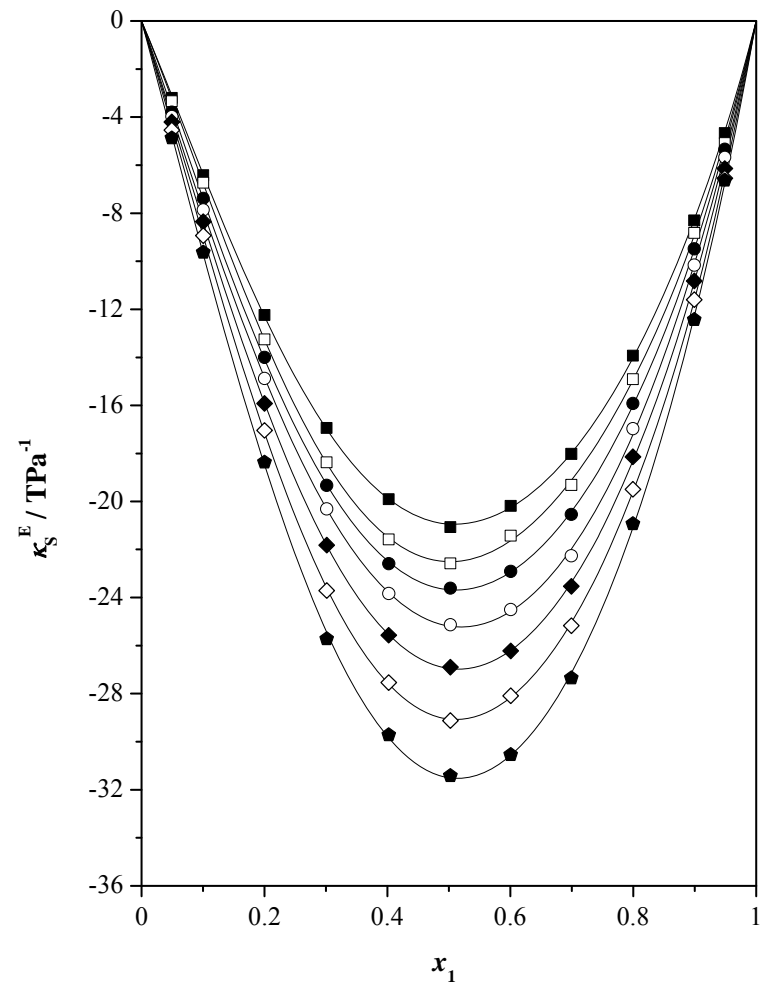

Excess isentropic compressibilities, $\kappa_{S}^{E}$, for 1-chloro-2-methylpropane + butyl ethyl ether as a function of mole fraction, $x_{1}:(\boldsymbol{\square}) T=283.15 \mathrm{~K}$, ( $\left.\square\right) T=288.15 \mathrm{~K}$, (○) $T=293.15 \mathrm{~K}$, (○) $T=$ $298.15 \mathrm{~K},(\diamond) T=303.15 \mathrm{~K},(\diamond) T=308.15 \mathrm{~K},(\bullet) T=313.15 \mathrm{~K} ;(-)$ Redlich-Kister equation. 
Fig. 4

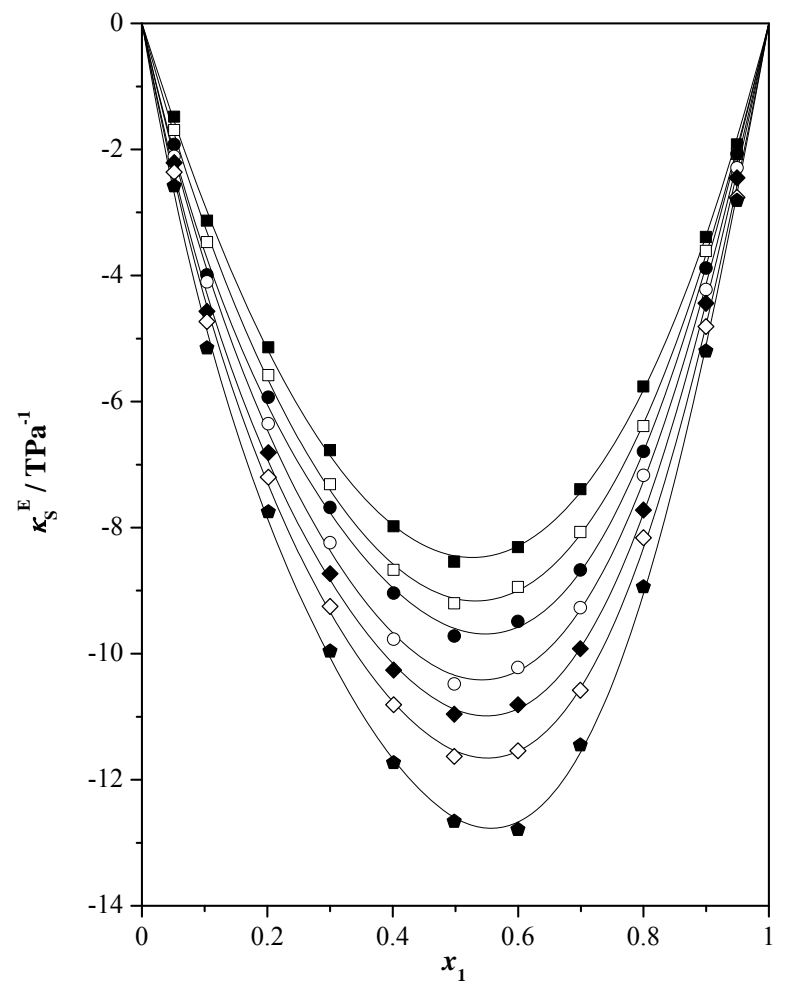

Excess isentropic compressibilities, $\kappa_{S}^{E}$, for 2-chloro-2-methylpropane + butyl ethyl ether as a function of mole fraction, $x_{1}:(\square) T=283.15 \mathrm{~K}$, ( $\left.\square\right) T=288.15 \mathrm{~K},(\bullet) T=293.15 \mathrm{~K}$, (○) $T=$ $298.15 \mathrm{~K},(\diamond) T=303.15 \mathrm{~K},(\diamond) T=308.15 \mathrm{~K},(\bullet) T=313.15 \mathrm{~K} ;(-)$ Redlich-Kister equation. 
Fig. 5

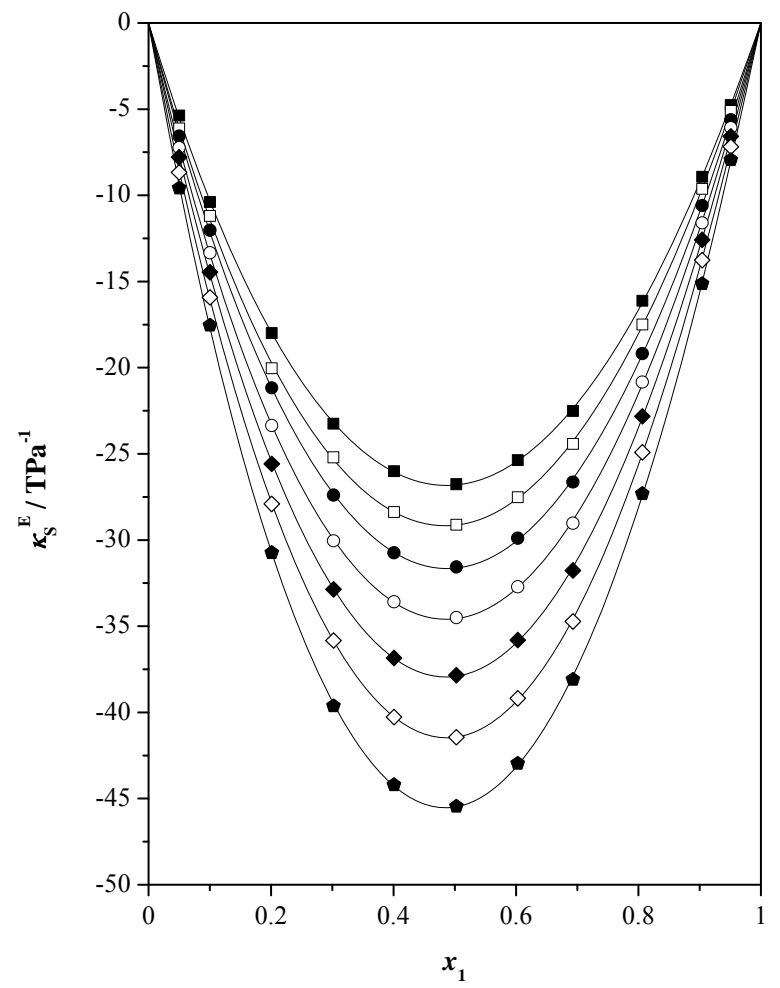

Excess isentropic compressibilities, $\kappa_{S}^{E}$, for 1-chlorobutane + methyl tert-butyl ether as a function of mole fraction, $x_{1}:(\square) T=283.15 \mathrm{~K},(\square) T=288.15 \mathrm{~K},(\bullet) T=293.15 \mathrm{~K}$, (○) $T=$ $298.15 \mathrm{~K},(\diamond) T=303.15 \mathrm{~K},(\diamond) T=308.15 \mathrm{~K},(\bullet) T=313.15 \mathrm{~K}$; (一) Redlich-Kister equation. 
Fig. 6

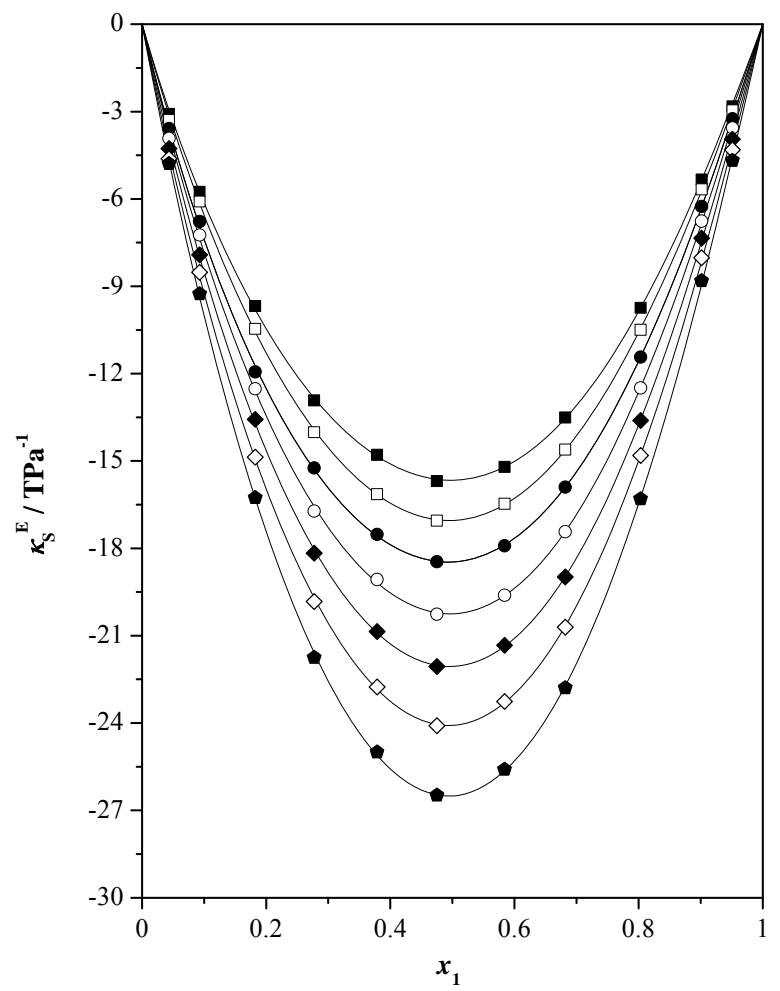

Excess isentropic compressibilities, $\kappa_{S}^{E}$, for 2-chlorobutane + methyl tert-butyl ether as a function of mole fraction, $x_{1}:(\square) T=283.15 \mathrm{~K},(\square) T=288.15 \mathrm{~K},(\bullet) T=293.15 \mathrm{~K}$, (○) $T=$ $298.15 \mathrm{~K},(\diamond) T=303.15 \mathrm{~K},(\diamond) T=308.15 \mathrm{~K},(\bullet) T=313.15 \mathrm{~K} ;(-)$ Redlich-Kister equation. 
Fig. 7

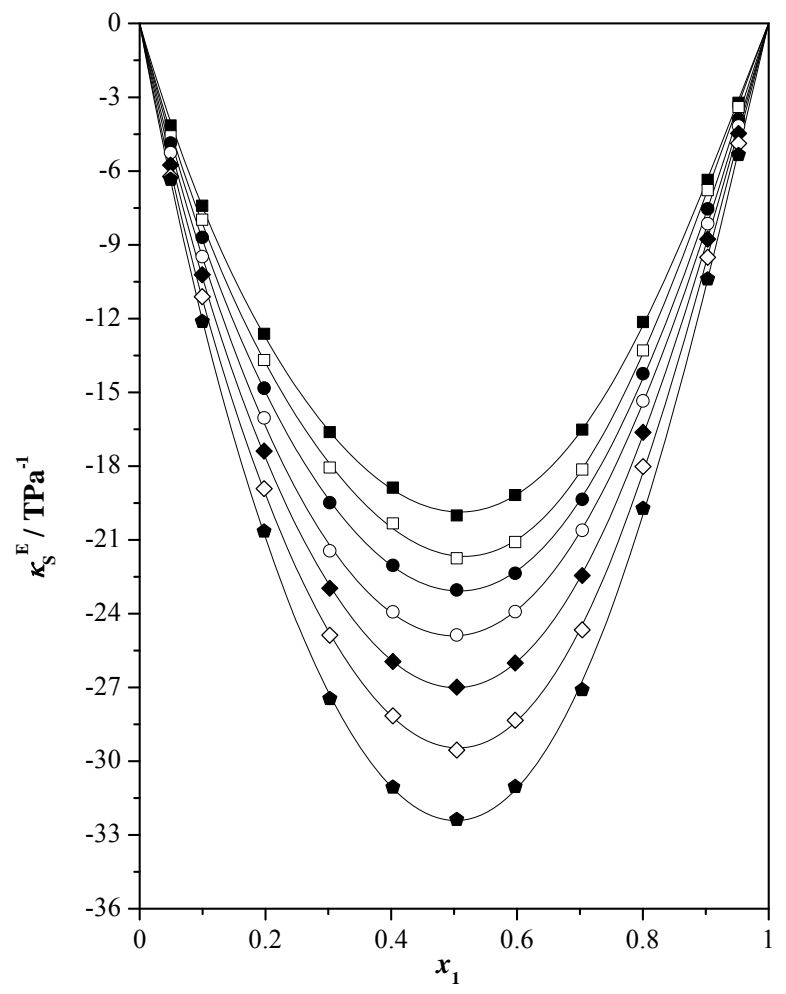

Excess isentropic compressibilities, $\kappa_{S}^{E}$, for 1-chloro-2-methylpropane + methyl tert-butyl ether as a function of mole fraction, $x_{1}:(\square) T=283.15 \mathrm{~K},(\square) T=288.15 \mathrm{~K},(\bullet) T=293.15 \mathrm{~K}$, (○) $T=298.15 \mathrm{~K},(\diamond) T=303.15 \mathrm{~K},(\diamond) T=308.15 \mathrm{~K},(\bullet) T=313.15 \mathrm{~K} ;(-)$ Redlich-Kister equation. 
Fig. 8

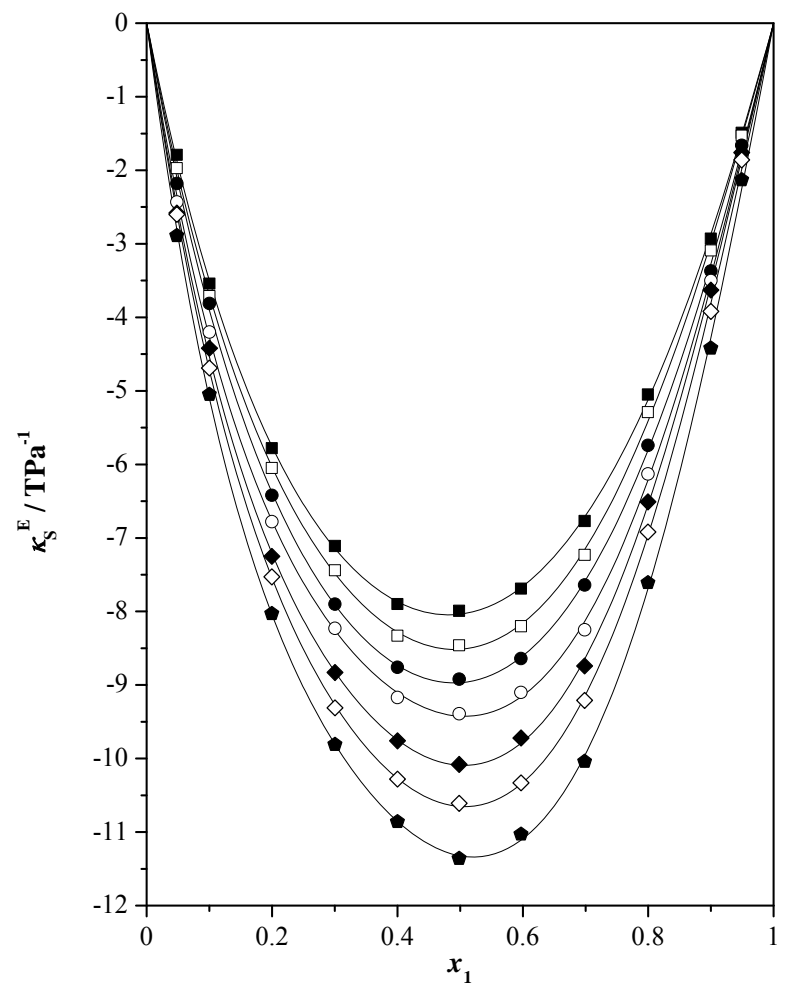

Excess isentropic compressibilities, $\kappa_{S}^{E}$, for 2-chloro-2-methylpropane + methyl tert-butyl ether as a function of mole fraction, $x_{1}:(\square) T=283.15 \mathrm{~K},(\square) T=288.15 \mathrm{~K},(\bullet) T=293.15 \mathrm{~K}$, (○) $T=298.15 \mathrm{~K},(\diamond) T=303.15 \mathrm{~K},(\diamond) T=308.15 \mathrm{~K},(\bullet) T=313.15 \mathrm{~K} ;(-)$ Redlich-Kister equation. 
Fig. 9

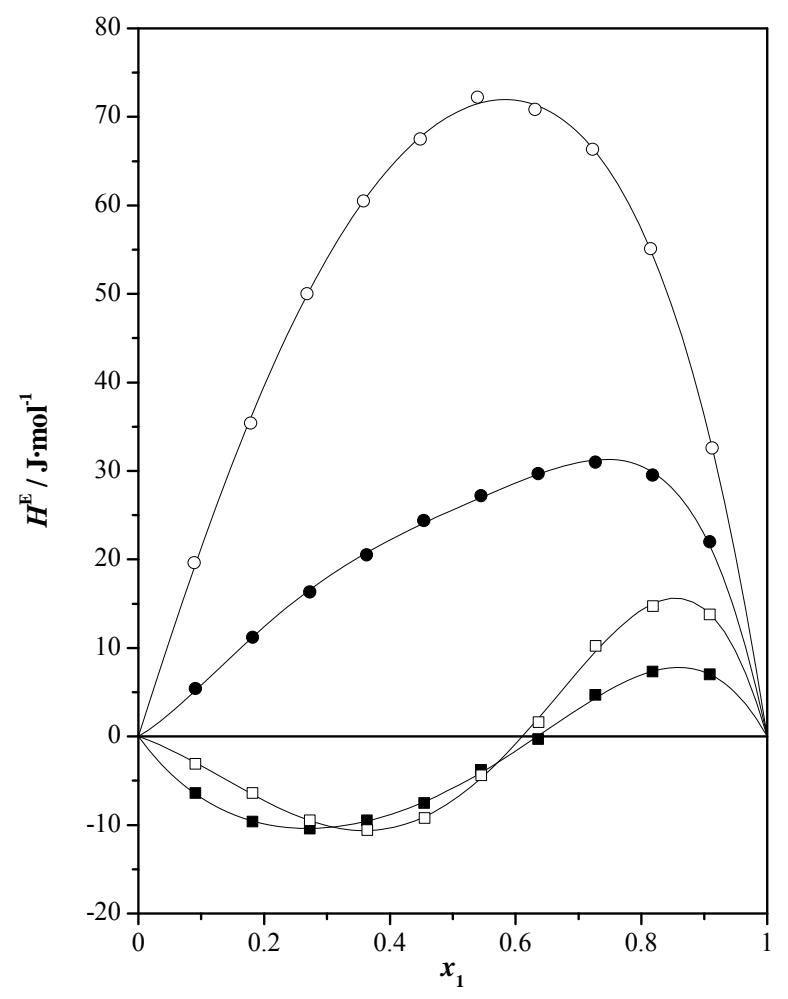

Excess enthalpies, $H^{\mathrm{E}}$, for isomeric chlorobutane + butyl ethyl ether at $T=298.15 \mathrm{~K}$ as a function of mole fraction, $x_{1}$ : (口) 1-chlorobutane; (O) 2-chlorobutane; ( $\square$ ) 1-chloro-2methylpropane; $(\bigcirc)$ 2-chloro-2-methylpropane; $(-)$ Redlich-Kister equation. 
Fig. 10

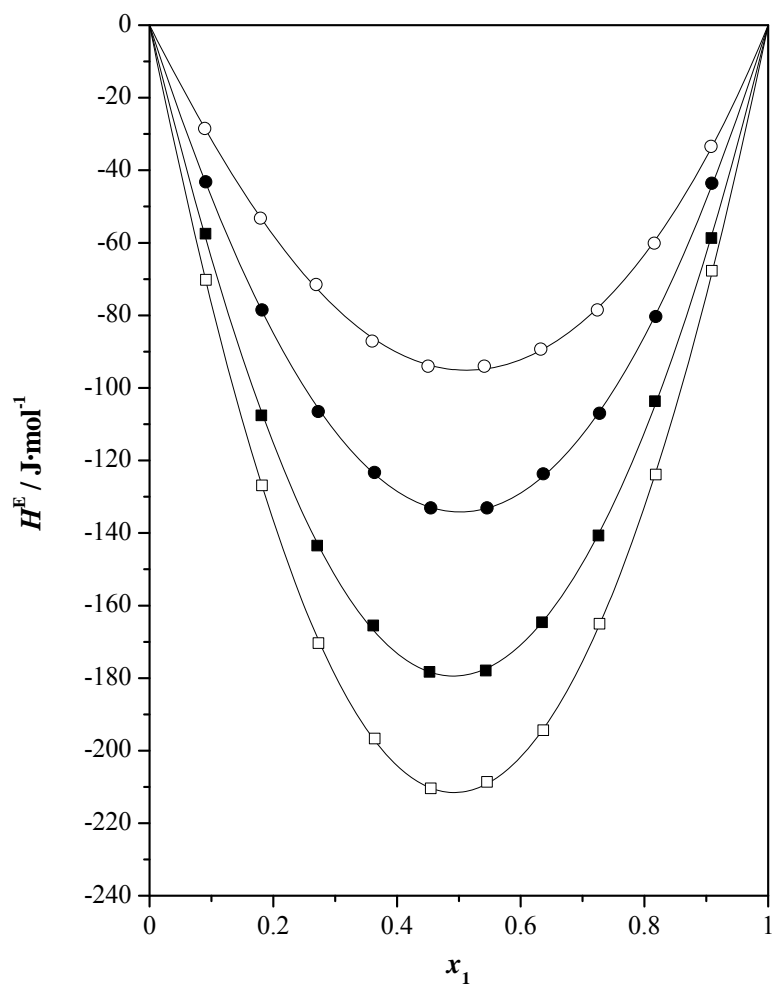

Excess enthalpies, $H^{\mathrm{E}}$, for isomeric chlorobutane + methyl tert-butyl ether at $T=298.15 \mathrm{~K}$ as a function of mole fraction, $x_{1}$ : (口) 1-chlorobutane; (ல) 2-chlorobutane; ( $\square$ ) 1-chloro-2methylpropane; $(\bigcirc)$ 2-chloro-2-methylpropane; $(-)$ Redlich-Kister equation. 